\title{
Design and fabrication of a prototype solar tapping device
}

\author{
Anirudh N. Kulkarni ${ }^{1}$, B. Arjun Bhat $^{1}$, Chinmay D. Sastry ${ }^{1}$, and Dr. K. S. Sridhar ${ }^{2}$ \\ ${ }^{1}$ Mechanical Engineering, PES University, Bengaluru, India \\ ${ }^{2}$ Department of Mechanical Engineering, PES University, Bengaluru, India
}

\begin{abstract}
Fossil fuel based energy sources are most commonly used because of the ease of availability and affordability, but have many long lasting negative effects, due to which a shift must be made to renewable and clean energy sources such as solar energy. Efficiency of the photovoltaic cells have increased by $1 \%$ every ten months, resulting in reduced costs and increased number of users. The project will explore the design and fabrication of an improved and efficient solar tapping device. An effort will be made to incorporate an architectural concept called "barrel vault" that is primarily used for natural lighting, over a Fresnel lens plate setup to act as a collector. The tracking mechanism incorporated is unique and can be modified to either have single axis or dual axis tracking depending on the requirements. Keeping in mind the environment and cost, an attempt has been made to fabricate the product with higher energy conversion rate, at an affordable cost using the above techniques. Upon analysing the results, the conclusion could be drawn that there was an increase in conversion rate of up to twice the traditional setup.
\end{abstract}

\section{Introduction}

There are primarily 2 classes of photovoltaics, or solar setups, based on concentration and solar cells, namely, conventional photovoltaics, that employ simple single junction solar cells, generally without any concentration or tracking mechanisms, and Concentrated Photovoltaics (CPV), that employ concentration setups along with multi junction solar cells [1].

Given that multi junction cells are costly and not mass produced everywhere, our setup attempts to use concentration methods on single junction PV cells that can also be a viable option for multi junction cells, essentially placing our solution between conventional PVs and CPVs.

Employing a barrel vault collection architecture that isn't fully used in solar devices, this allows for near- $180^{\circ}$ solar collection whilst not risking heating of the panel. The flat sides of the barrel vault have been left open allow for ventilation.

Beneath our barrel vault is a trapezoidal structure with a Fresnel lens plate [2] top, which is a plate with multiple small Fresnel lenses arranged on it, placed at about $80 \mathrm{~mm}$ above the solar panel, which is the approximate focal length of the Fresnel lenses used. This allows for multiple concentration points. The trapezoidal glass panes structure allows for uniform lighting above the panel when the sun is oblique to the panel and prevents shadow formation.

Dual axis tracking [3], while being more efficient than single axis tracking [4], can cause run time issues where the tracking algorithm in one direction interferes with the other. Hence, a single axis tracking algorithm from East to West has been implemented, essentially following the sun from sunrise to sunset. The barrel vault takes care of the summer to winter sun shift and is inclined to South by default.

\section{Methodology}

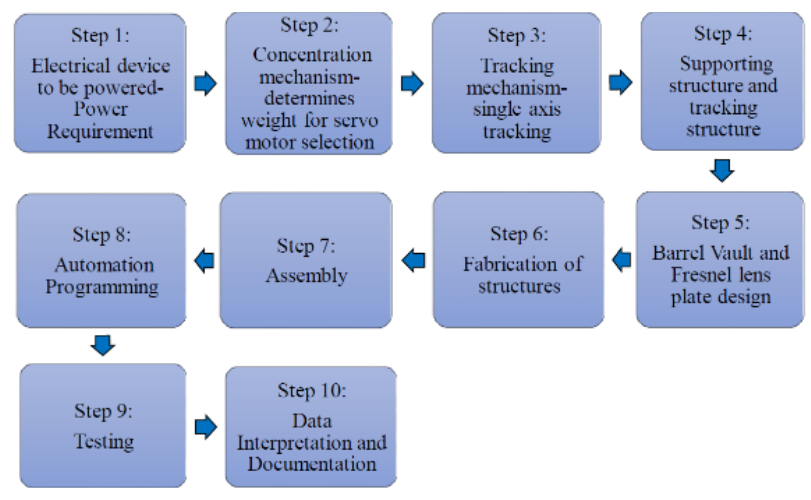

Fig. 1. Methodology Flowchart

- $\quad$ Power Requirement: $10 \mathrm{~W}$ panel for a DC bulb and a fan

- Concentration Mechanism:

a. Fresnel lens plate: 20 plano-convex lenses, $80 \mathrm{~mm}$ focal length, producing a concentration area of $30 \times 25 \mathrm{~mm}^{2}$ per lens.

b. Collection setup: Barrel Vault archway consisting of a total 6 transparent panels, around $310 \mathrm{~mm}$ diameter, of $81 \mathrm{~mm}$ arc length per panel.

c. Trapezoidal box of transparent planes covering the distance between barrel vault and glass panel.

- Tracking: Programmed to follow the sun from 8 am to $4 \mathrm{pm}$, moving at $15^{\circ}$ per hour 
- $\quad$ Support Structure Analysis: Analysed with a load of $30 \mathrm{kgs}$ put on the structure, corresponding to the weight of a full size solar panel and concentration mechanism. Modelled in Solidworks and analysed in ANSYS.

- Barrel vault design:

Diameter of barrel vault $=310 \mathrm{~mm}$

Total angle of barrel vault $=180^{\circ}$

Total number of glass panes $=6$

Angle of each glass pane $=180^{\circ} \div 6=30^{\circ}$

Using arc length formula $l=r * \theta$

$l=310 *\left(30^{\circ} * \pi \div 180\right)=81.158 \mathrm{~mm}$

- Fabrication: PLA (Polylactic acid), a biodegradable and bioactive thermoplastic aliphatic polyester, obtained from renewable sources like corn starch, cassava roots or sugar cane, was used as raw material to 3D print the base structure.

- Assembly: Structure assembled as shown-

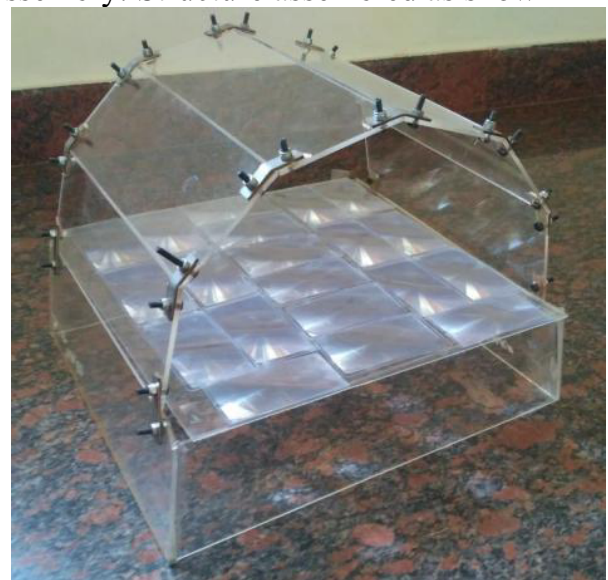

Fig. 2. Concentration setup
- Automation programming: Done on Arduino UNO IDE; programmed to move at $15^{\circ}$ per hour.

- Testing: Carried out for the following conditions, both with tracking and without tracking-

1. Fixed Solar Panel only

2. Panel with Fresnel Plate

3. Panel with Fresnel Plate and Barrel Vault

\section{Experimentation and results}

The one trial, testing was done taking, a solar panel setup, solar panel setup coupled with a Fresnel plane setup and finally the prototype that consisted of the panel coupled with both the Fresnel plane setup and the barrel vault setup. Readings were obtained without tracking the position of the sun and the data in Table 1 was obtained.

During this test, data for a basic panel, panel+ Fresnel setup and panel + Fresnel + Barrel setup, was compared. Peak current and voltage output was obtained for a basic solar panel as it works under both direct and diffuse sunlight, in contrast to the Fresnel setup that works only under direct sunlight. A steady output voltage was obtained for all the three setups. The setup involving the Fresnel setup had lower output voltage owing to shadow experienced by the panel in the current setup. The output current was maximum for the basic setup, but a low and steady value was obtained for the Fresnel setup. The current peaked at 12 noon when the sun was overhead and the sunrays were incident normally, for the final prototype. This was an indicator of the device working well with sun tracking when the rays would be incident normally.

Table 1. Test Results for complete setup without Tracking

\begin{tabular}{|c|c|c|c|c|c|c|c|}
\hline Date & $10-04-2018$ & \multicolumn{2}{|c|}{$\begin{array}{c}\text { SOLAR PANEL } \\
\text { WITHOUT TRACKING }\end{array}$} & $\begin{array}{c}\text { SOLAR PANEL+ FRESNEL } \\
\text { SETUP WITHOUT TRACKING }\end{array}$ & \multicolumn{2}{|c|}{$\begin{array}{c}\text { SOLAR PANEL+ } \\
\text { FRESEL+BARREL } \\
\text { WITHOUT TRACKING }\end{array}$} \\
\hline Time & Temperature (C) & $\begin{array}{c}\text { Voltage 1 } \\
(\mathrm{V})\end{array}$ & $\begin{array}{c}\text { Current } \\
(\mathrm{mA})\end{array}$ & Voltage 2(V) & Current 2 (mA) & $\begin{array}{c}\text { Voltage 3 (V) } \\
\text { Current 3 } \\
(\mathrm{mA})\end{array}$ \\
\hline $08: 00$ & 22 & 19 & 200 & 18.1 & 180 & 18.8 & 200 \\
\hline $09: 00$ & 24 & 19.9 & 300 & 19.5 & 250 & 18.4 & 250 \\
\hline $10: 00$ & 27 & 20.4 & 500 & 19.8 & 250 & 19.6 & 250 \\
\hline $11: 00$ & 29 & 20 & 520 & 19.6 & 270 & 19.3 & 270 \\
\hline $12: 00$ & 30 & 19.9 & 530 & 19.4 & 400 & 19.1 & 400 \\
\hline $13: 00$ & 31 & 19.8 & 520 & 19.4 & 400 & 19.1 & 300 \\
\hline $14: 00$ & 32 & 19.6 & 520 & 18.8 & 300 & 19.2 & 250 \\
\hline $15: 00$ & 32 & 19.6 & 260 & 19.3 & 240 & 18.9 & 250 \\
\hline $16: 00$ & 31 & 19.2 & 220 & 19 & 200 & 18.9 & 220 \\
\hline
\end{tabular}




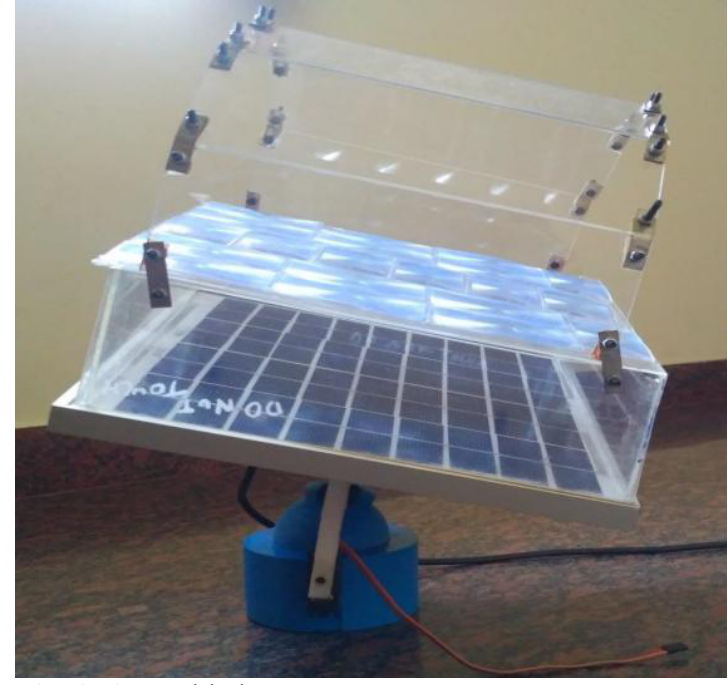

Fig. 3. Assembled Prototype

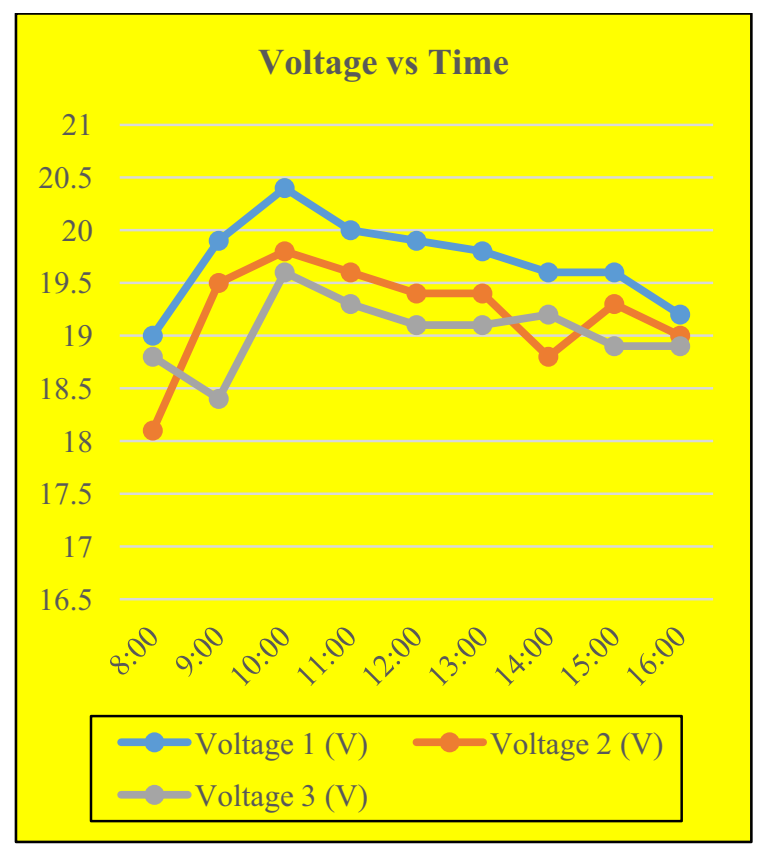

Fig 4. Graph of Voltage (V) vs Time of Day

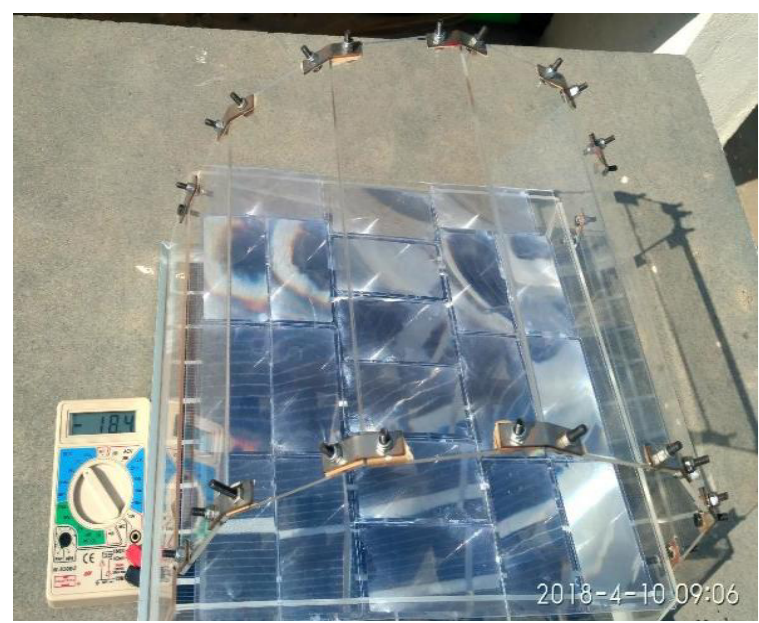

Fig. 5. Prototype and testing apparatus

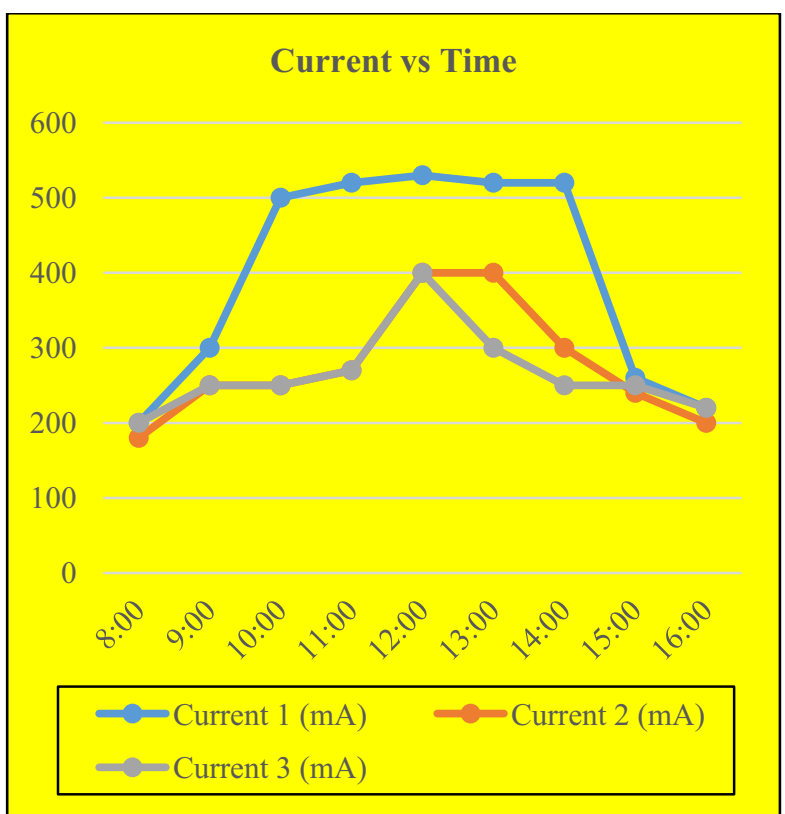

Fig 6. Graph of Current (mA) vs Time of Day

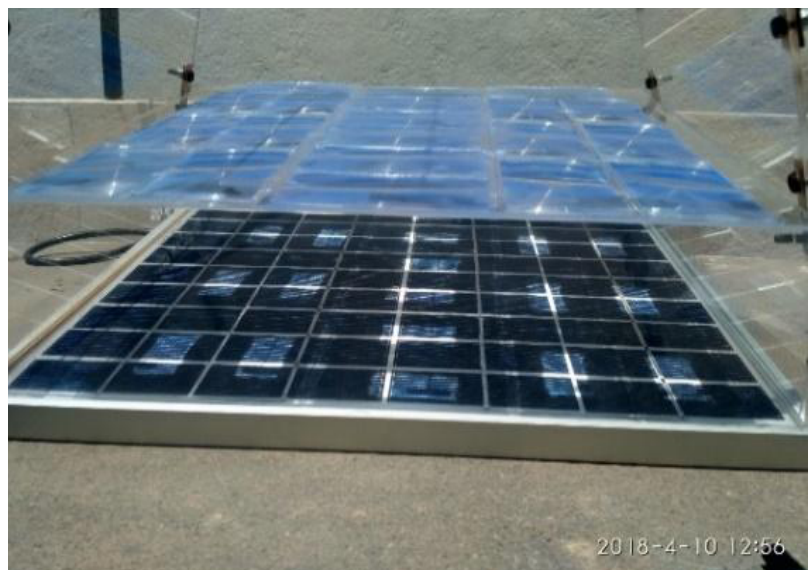

Fig. 7. Close up view of concentration zones on the panel

Fig. 5 shows concentrated sunlight being incident on the panel at 12:56 PM. Each of the concentrated sunlight rectangles have an area of $8.75 \mathrm{~cm}^{2}$ and there is a total of 20 such rectangles, resulting in a total area of $175 \mathrm{~cm}^{2}$. Each panel rectangle area is $10 \mathrm{~cm}^{2}$ and total area of the panel is $720 \mathrm{~cm}^{2}$ with 72 rectangles. Therefore, for just $24.3 \%$ of the panel receiving concentrated sunlight at normal incidence, the output power is $5.73 \mathrm{~W}$. Placing the solar cells strategically so that all of them receive concentrated sunlight could result in a power that is 4 times the previous value of $22.9 \mathrm{~W}$. However, a basic panel setup exposed to sunlight at the same time, resulted in a power output of $10.49 \mathrm{~W}$. This clearly indicates a higher energy conversion rate per cell for a combined Fresnel and barrel setup.

\section{Conclusions}

The prototype was successfully designed and fabricated. With our setup of barrel vault on top of the panel with the Fresnel lens to concentrate the sunlight, we could observe that only $24.3 \%$ of the panel received concentrated sunlight at normal incidence, with output 
power being $5.73 \mathrm{~W}$. This would mean more output power per exposed area of the panel. For this setup, if the solar cells were strategically placed to receive the concentrated sunlight, the power output could rise to 4 times the current value. Following are some of the advantages of the proposed device over the basic solar setup: 1) The device has a higher energy conversion rate for the same panel area in comparison to the basic setup. 2) Lesser space required for the installation of the device for the same power output. Considering a higher energy conversion rate per cell by the incorporation of the several innovative technologies, pointed to the fact of the device being a success. Some of the aspects that can be worked on, to improve the existing device would include the following: 1) Switching to multi-junction solar cells in place of single junction cells, could result in higher output because of better temperature characteristics of the former. 2) Accurate placement of the solar cells to receive direct concentrated sunlight from the Fresnel plane. 3) Optimization and mathematical modeling of the radius of curvature of the barrel vault setup to account for the seasonal changes. 4) The current setup utilizes only single axis tracking and having dual axis tracking could improve the efficiency.
Considering a higher energy conversion rate per cell by the incorporation of the several innovative technologies, pointed to the fact of the device being a success.

\section{Acknowledgement}

The authors would like to thank the management and the Department of Mechanical Engineering of PES University for their continued support in the completion of this research.

\section{References}

1. T.S. Viana, R. Ruther, F.R. Martins and E.B. Pereria, Sol. En., vol. 85, pp. 486-495, (2011)

2. J.-N. Juang and R. Radharamanan, American Society for Engineering Education, (2014)

3. T.-S. Zhan, W.-M. Lin, M.-H. Tsai and G.-S. Wang, Computer Software and Applications Conference (Kyoto, Japan, 2013)

4. D. S., R. R., P. A., R. Dhanabal, IJESIT, 2 (2013) 